\title{
Characterization of Radiolucent and Electro-Magnetic Properties of Polymer PEDOT: PSS for Radiological Applications
}

\author{
Laura Guérin1, Christophe Loyez ${ }^{2}$, Kamel Guerchouche1, Fouad Maaloul1 \\ ${ }^{1}$ Biomediqa, 99C Rue Parmentier, Villeneuve d'Ascq, France \\ ${ }^{2}$ CNRS, UMR 8520 IEMN, IRCICA, Lille, France \\ Email: laura.guerin@biomediqa.com
}

How to cite this paper: Guérin, L., Loyez, C., Guerchouche, K. and Maaloul, F. (2017) Characterization of Radiolucent and Electro-Magnetic Properties of Polymer PEDOT: PSS for Radiological Applications. Open Journal of Organic Polymer Materials, 7, 17-28. https://doi.org/10.4236/ojopm.2017.72002

Received: March 14, 2017

Accepted: April 15, 2017

Published: April 18, 2017

Copyright (c) 2017 by authors and Scientific Research Publishing Inc. This work is licensed under the Creative Commons Attribution International License (CC BY 4.0). http://creativecommons.org/licenses/by/4.0/

\section{Open Access}

\begin{abstract}
The deployment of wireless technologies in the radiology field requires development of radiolucent materials allowing wireless communication. In this paper, both the radiolucency (X-rays) and Electro-Magnetic (EM) properties of the conductive polymer poly(3,4-ethylenedioxythiophene) polystyrene sulfonate (PEDOT:PSS) are investigated to address wireless applications in the field of the radiology. A model was developed to predict the PEDOT:PSS performance and assess the interest of this material in terms of radiolucency characteristics and Electro Magnetic (EM) radiation efficiency. A PEDOT:PSS antenna operating in the Radio-Frequency Identification (RFID) Ultra High Frequency (UHF) band, was realized to experimentally validate abilities of PEDOT:PSS. These performances are compared with those produced by a similar antenna made of conventional materials such as copper. Experimental results validate the numerical model of the designed structure. This paper demonstrates the potential of polymer materials in the field of $\mathrm{x}$-ray imaging.
\end{abstract}

\section{Keywords}

PEDOT:PSS, Radiology, Radiolucency, Wireless Technologies

\section{Introduction}

Wireless technologies are more and more involved in medical fields in general and radiology applications in particular [1]: further and further wireless technologies are involved in hospitals, to record vital signs for example [2] [3] [4]. They can allow patient monitoring at his bedside or during transport. There are no disconnection and reconnection of cables and thus no interruption of patient 
monitoring. They prevent heavy cable equipment for heart constants on newborns [5]. In the medical device industry, the devices that require more and more advances are often constrained and limited by their construction materials. Mechanical, structural and conductive components are usually made of metals such as aluminum, titanium and copper, but these materials are radiopaque to $\mathrm{x}$-rays. A surgeon cannot properly operate during an interventional radiology procedure when a metallic device obstructs the image. A wide variety of applications like head supports or monitoring electrodes needs both the physical and chemical properties of metals and radiolucent properties [6]. Today, the majority of hospitalized patients need x-rays or a scan: wireless radiolucent systems may find application in radiology. The radiolucency is an important property of a sensor in radiology to avoid image artifacts caused by the x-ray radiation used in combination with dense materials. Images with errors have to be avoided: it interferes with accurate interpretation and diagnosis. Today, wireless radiolucent sensors are systems of interest to assist the medical personal.

The conductive materials commonly used in RFID are copper and aluminum but are a priori radiopaque. Alternative solutions should be considered. The motivation of this paper is to overcome this limitation by introducing novel technologies: the best known Conductive Polymer (CP), PEDOT:PSS, which exhibits both transparency characteristics in the visible and electrical conductivity. PEDOT:PSS or poly(3,4-ethylenedioxythiophene) polystyrene sulfonate is a polymer mixture of two ionomers. CPs are already used in photovoltaic cells or computer displays, and are now finding applications in the field of biomaterials, e.g. in neural electrodes [7].

The goal of this study is to propose a radiolucent antenna that can be used in the medical field, especially in radiology. This RFID antenna could be associated with sensors to monitor the patient and record vital signs. This wireless monitoring would provide a continuous follow-up of the patient during his hospitalization, including in the operating room, and without disturbing imaging procedures. This antenna could also be used for problems of patient's identification.

The first part of this paper deals with the material and methods. First, the wireless device has been modeled to quantify the radiolucency behavior to X-ray in the case of PEDOT:PSS in comparison with the conventional materials (copper). Then, the radiolucency of the PEDOT:PSS wireless device has been experimentally tested (experimental set-up) and the model has been validated (radiography). At this step, the electromagnetic (EM) radiation efficiency of the wireless device has been modeled and compared to that of conventional materials.

\section{Material and Methods}

For this study, we have considered a wireless device operating in the industrial, scientific and medical (ISM) radio bands (Ultra High Frequency - UHF: 860 $960 \mathrm{MHz}$ ). This wireless device is based on a Radio-Frequency Identification 


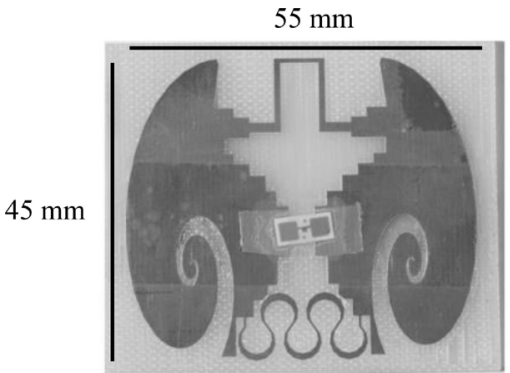

(a)

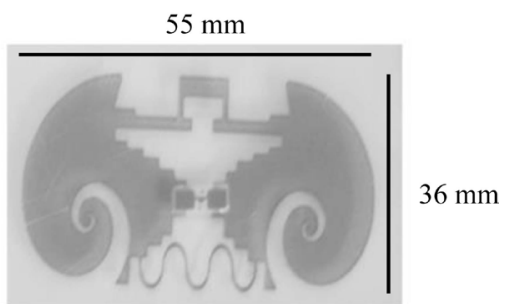

(b)

Figure 1. (a) Copper antenna $(55 \times 45 \mathrm{~mm})$ and (b) PEDOT:PSS antenna $(55 \times 36 \mathrm{~mm})$. The dimensions are optimized on each antenna to maximize the EM efficiency.

(RFID) topology which mainly consists of a broadband antenna. The RFID antenna is the critical element for a good performance of a RFID tag, the chip has a limited sensitivity. With same chip performance, the range of an UHF RFID tag may vary from by a factor two depending on antenna performance. Two versions are considered: the first one (Figure $1(\mathrm{a})$ ) has been achieved using conventional materials such as copper and epoxy. The second one involves exclusively biocompatible materials which are potentially radiolucent (glass as a substrate and PEDOT:PSS as a conductor) as depicted by Figure 1(b). PEDOT:PSS represents a promising alternative to metallic conductors, in terms of biocompatibility. We use a Clevios S V4 product of Heraeus Company that is compatible with screen printing deposition. The copper antenna has a thickness of $35 \mu \mathrm{m}$ and that of PEDOT:PSS of $6 \mu \mathrm{m}$, these thicknesses are those achievable by conventional industrial processes. In both cases, the same design of the antenna is used but the shape and size of the copper and PEDOT:PSS antennas are slightly different as they have been adapted to optimize and maximize electromagnetic efficiency according to Figure 1.

\subsection{Model of the Radiolucency Behavior of PEDOT:PSS}

The radiology device has been modeled. A model involving a vast amount of Monte-Carlo simulations has been developed to reproduce the performance of an $\mathrm{x}$-ray device and to simulate the particle tracks and interactions with materials, according to probability density distributions implied by particle and material properties [8]. The model is based on the Allura Xper system from Philips, dedicated to cardiovascular interventions [9]. The system consists of an $\mathrm{x}$-ray tube and a flat panel detector that can be translated in three dimensions.

$\mathrm{X}$-rays are produced in $\mathrm{x}$-ray tubes. Electrons are emitted from a cathode (filament heated by the passage of an electric current) and are accelerated by a high potential difference toward a target consisting of an anode metal. X-rays are emitted by the target by two mechanisms:

-The braking of the electrons on the target creates a continuous radiation (bremss trahlung).

-The accelerated electrons have sufficient energy to excite certain of the atoms of the target, disrupting their internal electronic layers. These excited atoms emit 
$\mathrm{x}$-rays returning to their ground state.

A small portion, about $1 \%$ of the kinetic energy lost by the electrons radiated as $\mathrm{X}$-rays, $99 \%$ is converted into heat energy.

The intensity and quality of the radiation emerging from an $\mathrm{x}$-ray tube finally are primarily a function of:

-High voltage potentials between $40 \mathrm{kV}$ and $150 \mathrm{kV}$;

-Tube current up to $1000 \mathrm{~mA}$;

-Exposure time;

-Inherent and filtrations that increase the average energy of $\mathrm{X}$ multi-energy beam and remove low energy photons, unable to reach the detector, but with a sufficient energy to reach and irradiate the patient unnecessarily $(2.5 \mathrm{~mm}+1.0$ $\mathrm{mm}$ of $\mathrm{Al}+0.4 \mathrm{~mm}$ of $\mathrm{Cu})$.

We use the GATE (Gated Application Tomography Emission) platform which has received a lot of interest in the medical physics research community because of a number of attractive features [10]. GATE is built on an open source of the GEANT4 Monte Carlo code, software developed by the high energy physics community to design its very large detectors [11] [12]. A macro mechanism is integrated into the code to reproduce simple or sophisticated experimental settings.

Geometry of the x-ray device is designed according to the manufacturer data. In order to obtain simulated images, the detector is modeled as a CsI flat plate. It is positioned in line with the radiation beam's central axis and the distance between the detector and the x-ray tube is of $100 \mathrm{~cm}$. Still using the GATE platform, both the antennas are modeled by considering their materials, the dimensions used are those of PEDOT:PSS antenna (Figure 2).

A complete $\mathrm{x}$-ray tube simulation is a very time-consuming task because a large number of photon events are involved to obtain an energy distribution of the beam (spectra). To overcome this issue, it is possible to use many computing theoretical $\mathrm{x}$-ray spectra that have been developed which are in adequacy with the use of GATE. We choose to base our x-ray prediction model on the software program SpekCalc (Institute of Cancer Research in London, UK) [13] [14] [15]. SpekCalc calculates $\mathrm{x}$-ray spectra from a tungsten anode $\mathrm{x}$-ray tube. Input parameters are peak voltage, anode angle, equivalent aluminum filtration ... The

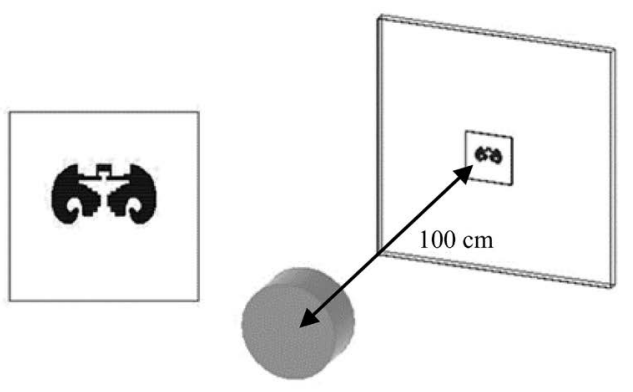

(a)

(b)

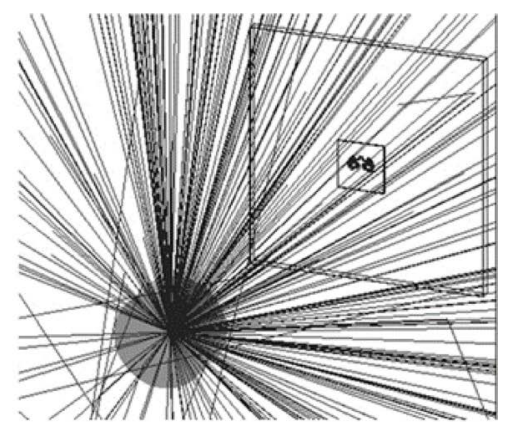

(c)

Figure 2. (a) Antenna model, (b) Antenna and x-ray tube, (c) Simulation. 
Monte-Carlo simulations are extremely consuming in computing time, the simulation execution on a single station is critical if the results are expected with realistic time. The Virtual Imaging Platform (VIP) is used to facilitate access to simulators and to the computing means and storage required for the simulation of medical images [16].

\subsection{Experimental Radiolucency of PEDOT:PSS and Validation of the Model}

Antennas are directly placed on the detector for radiography. This situation represents the worst case in terms of radiolucency. In a normal situation, the patient's presence and the examination table attenuate the $\mathrm{x}$-ray beam on the antenna and improve the radiolucency of the antenna. Projection radiography images were acquired considering the same irradiation geometry employed in obtaining the experimental images and according to the same image format. For each simulation, $10^{10}$ photons were emitted according to the $\mathrm{x}$-ray spectra, to reproduce as accurately as possible an $\mathrm{x}$-ray image taking into account the performance of the computer.

Studies dealing with radiolucency are most often confined to a visual comparison [17] [18] [19]. The visual estimate of the radiolucency or not of the antenna depends on the operator and is therefore much more subjective. Our model allows us to deepen the subject and also to compare the images numerically. It is here that a numerical analysis becomes interesting to characterize the radiolucency of a material: the grey level of the profile along a line (Figure 3 ) has been plotted for used images; these curves are normalized to the average of the grey values of pixels in the background of the image.

From an energy point of view, the impact of the presence of the antenna on the beam quality, and therefore on the quality of the produced image is also studied. For that, energy spectra are calculated after irradiation of the antenna and are studied. These spectra represent the number of photons detected by the detector as a function of their energy; they directly influence the properties of the

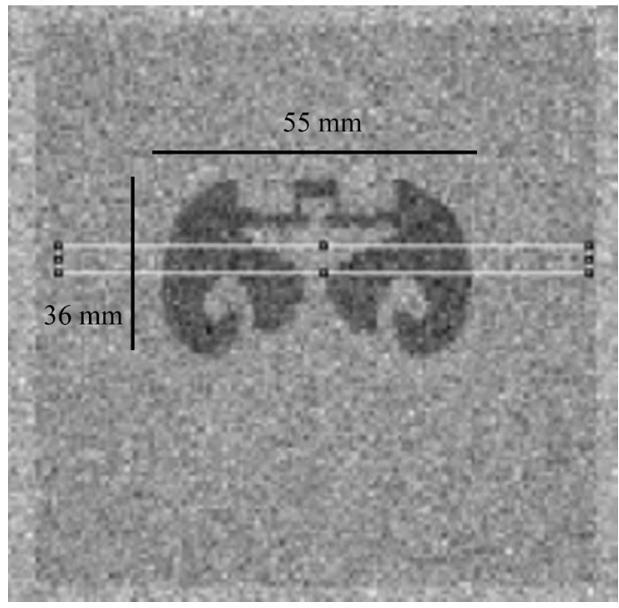

Figure 3. Region of interest along a line for the image profile. 
image. For easier reading of spectra, the antennas are modeled by plate of copper and PEDOT:PSS that cover the detector, with the same thickness as that used for the antennas. $10^{10}$ particles are simulated; the energy spectra are normalized by the total number of particles.

\subsection{Model of the EM Radiation Efficiency of PEDOT:PSS and Conventional Materials}

The numerical simulation allows us to estimate how the copper and PEDOT antennas radiate into space. The simulation procedure is to draw the $3 \mathrm{D}$ geometry of the simulated device, specifying the materials used for each element of the structure.

The CST software is dedicated to the simulation and design of RF circuits and electronic systems. This software uses finite differential elements to calculate electromagnetic fields in 3D. It gives among others, the values of the parameters of the structures, its radiation pattern and gain, which determines its radiative efficiency and then the reading distance. To model the PEDOT conducting structure we consider the film as a thin layer of a sheet conductivity simulated as a lossy material, with the temporal solver and an adaptive hexahedral mesh.

\section{Results}

\subsection{Visually and Numerically}

The images are first studied visually: experimental and simulated images are compared (Figure 4). The results obtained are those expected and provide results comparable to those obtained experimentally: it validates the model as first estimate of radiolucency, it reveals the radio-opacity of the copper antenna (35 $\mu \mathrm{m})$ and the radiolucency of the PEDOT:PSS antenna $(6 \mu \mathrm{m})$. On PEDOT:PSS

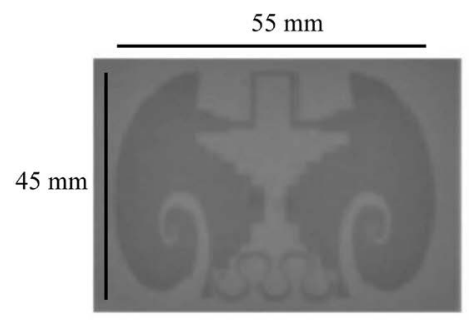

(a)

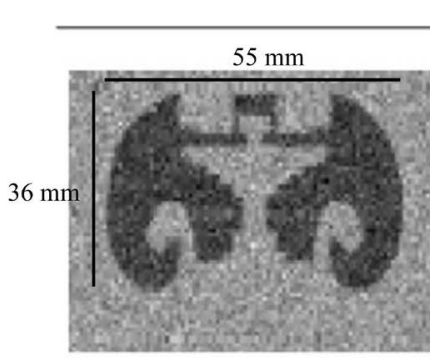

(c)

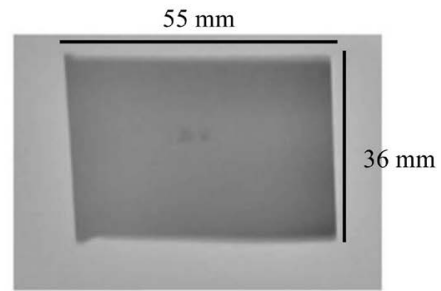

(b)

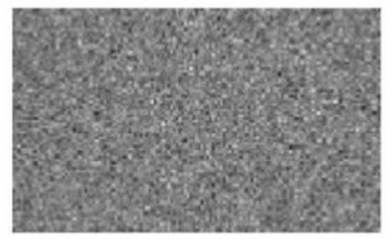

(d)

Figure 4. Visual comparison between model and radiography. (a) Copper radiography, (b) PEDOT:PSS radiography, (c) Copper simulation, (d) PEDOT:PSS simulation. 


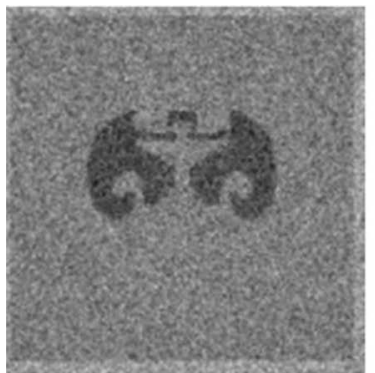

(a)

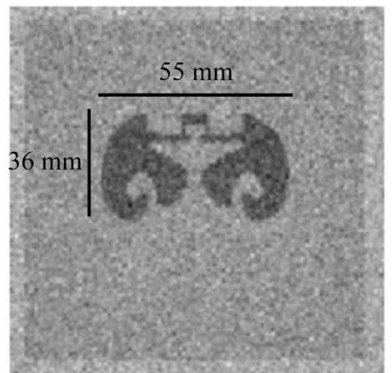

(b)

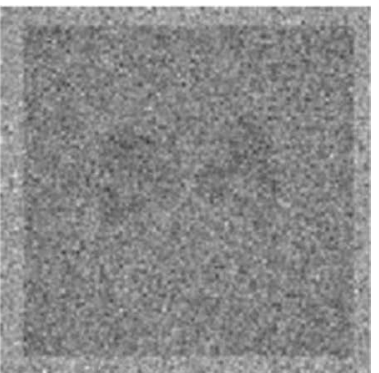

(c)

Figure 5. Radiolucency comparison of three copper thicknesses: (a) $35 \mu \mathrm{m}$ copper, (b) 18 $\mu \mathrm{m}$ copper and (c) $6 \mu \mathrm{m}$ copper.

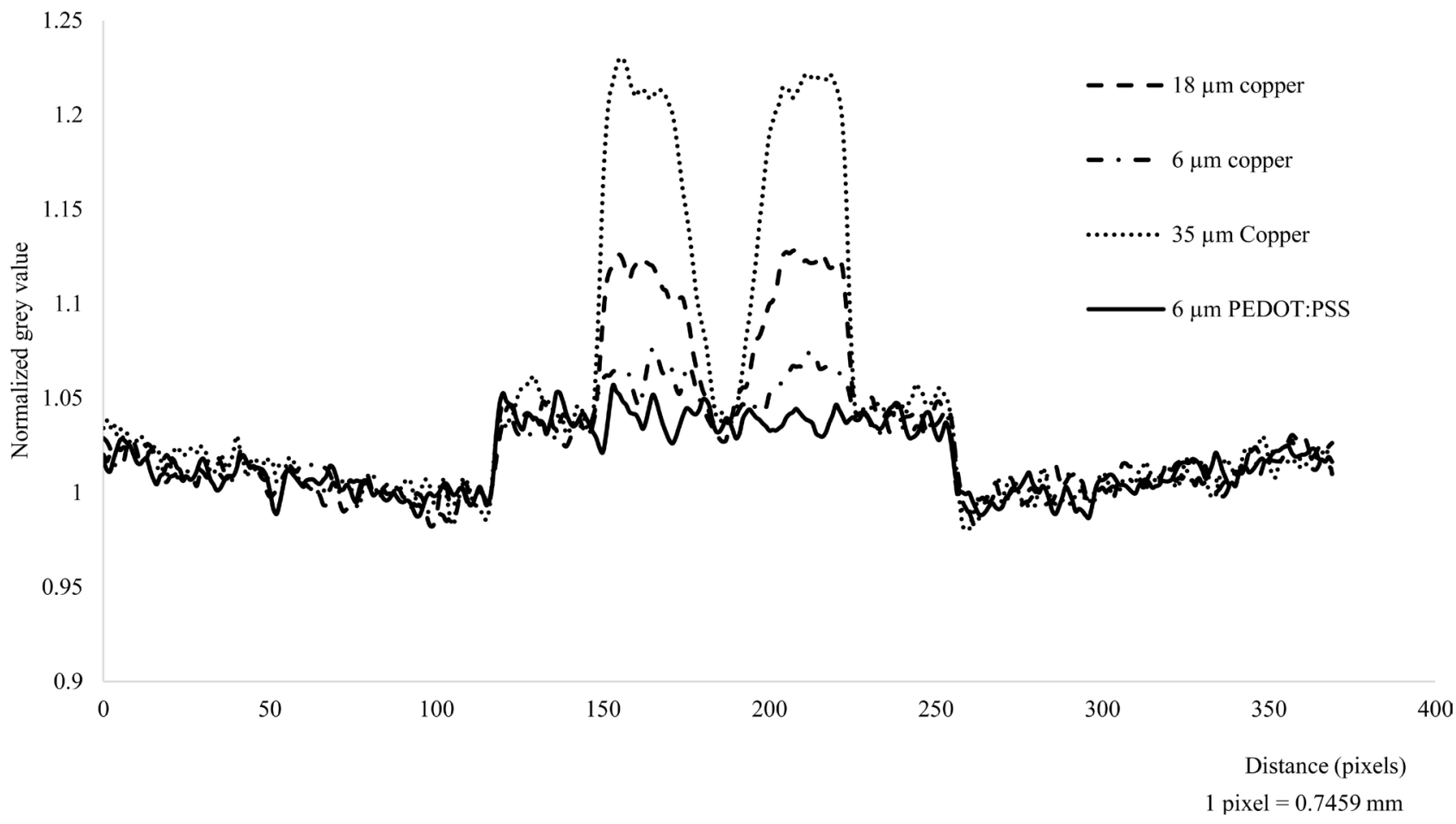

Figure 6. Profile along a horizontal line on the image.

radiography, only the packaging strap, which allows the antenna to be connected to the RFID chip, is visible. This packaging strap whose precise composition is unknown, is not simulated in our model. Other copper thicknesses (18 $\mu \mathrm{m}$ and 6 $\mu \mathrm{m})$ were simulated with the model in order to investigate the limits of the radiolucency of copper (Figure 5). The 18 micrometers antenna is clearly visible. The 6 micrometers copper antenna of the same thickness as the thickness of the PEDOT antenna looks like still visible on the image.

Figure 6 illustrates the grey level of the profile along a line and has been plotted for each of these images. It highlights the attenuation of different materials: air, glass, copper and PEDOT:PSS. In this graph, the greater the copper thickness is, the higher the grey value of pixels is. The curve representing the $18 \mathrm{mi}$ crometers antenna profile confirms the visual radiopacity previously presented. About the antenna of 6 micrometers, its profile is slightly above that of the glass, 
this explains its weak opacity in the image. Regarding the antenna PEDOT, profile in PEDOT and profile in glass have no difference which proves its radiolucency.

Figure 7 compares the energy of photons after the passage of 35 micrometers of copper, 6 micrometers of PEDOT and in the reference case where there is nothing (in the air). There is no influence from the antennas, whether copper or PEDOT:PSS. Figure 8 compares different copper thicknesses. Thick plate of 400

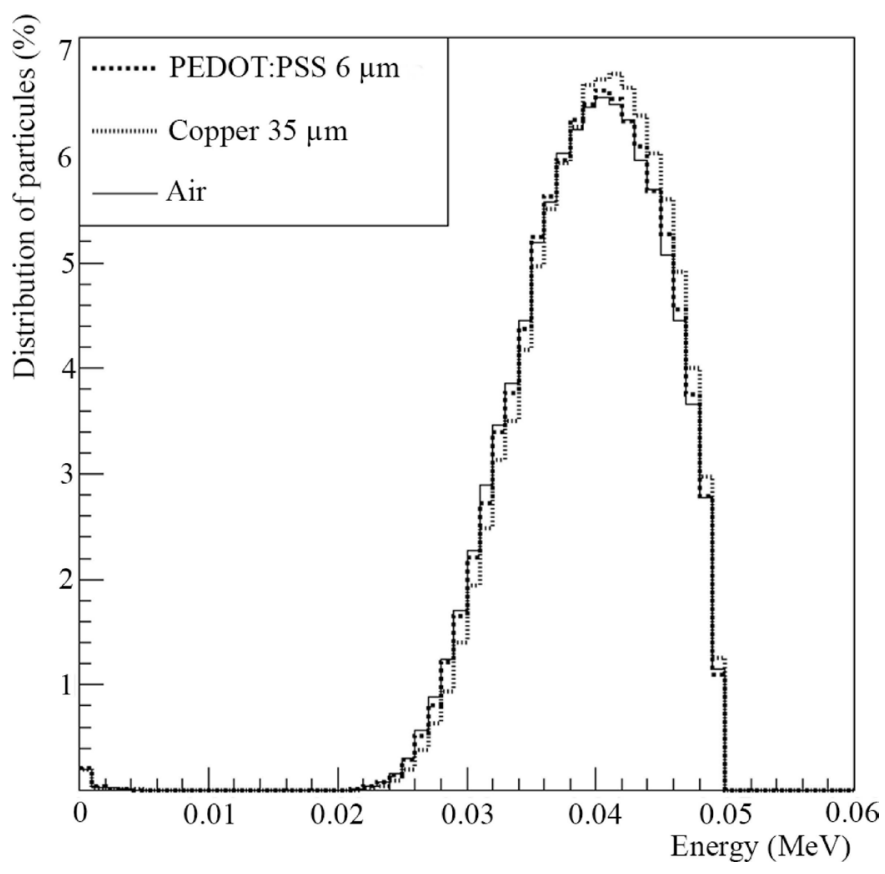

Figure 7. The impact of copper and PEDOT:PSS on the beam quality.

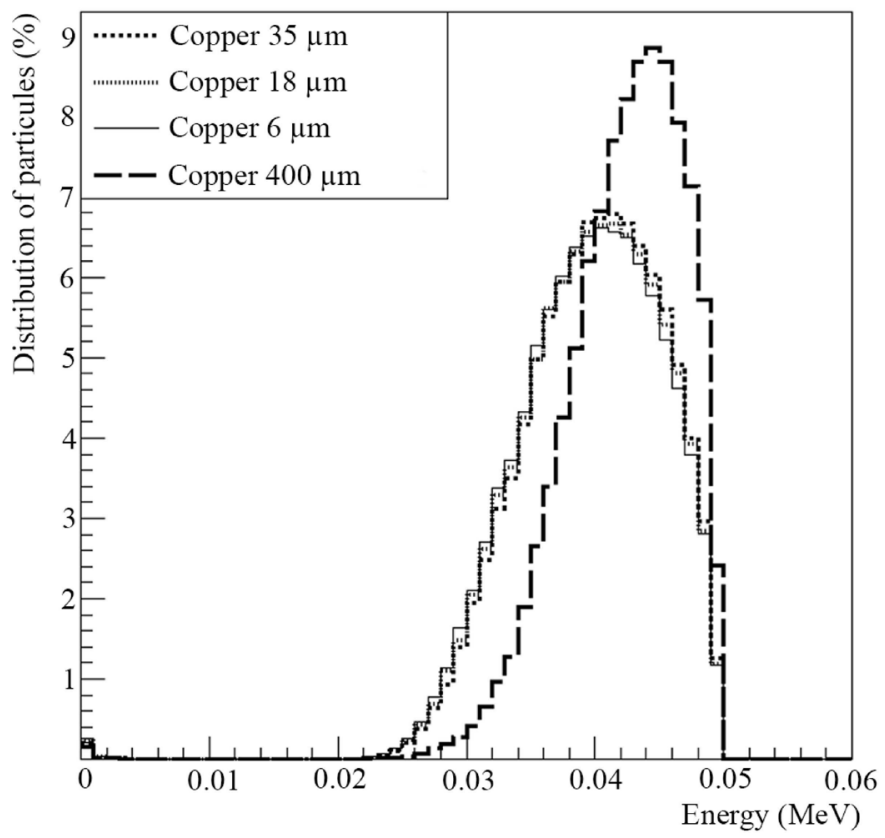

Figure 8. The impact of copper on the beam quality. 
micrometers of copper provides a benchmark for the medical physicist: this kind of plate is commonly used as a filter to harden the beam in radiology and reduce low energy photons that have no use. The spectra for thicknesses of 6,18 and 35 micrometers do not exhibit differences. Their radiopacity involves no impact at the energy level and therefore no impact on the quality image.

\subsection{Radiation Efficiency}

The copper based antenna conductivity is about $5.8 \times 10^{7} \mathrm{~S} / \mathrm{m}$ with a thickness of $35 \mu \mathrm{m}$ and that of the PEDOT antenna is around $1.5 \times 10^{4} \mathrm{~S} / \mathrm{m}$ with a thickness of $6 \mu \mathrm{m}$. Thicknesses are dependent on the processing method. $35 \mu \mathrm{m}$ copper thickness is a conventional industrial process. The deposited PEDOT:PSS thickness is small, beyond this thickness, the processing printing method cannot be directly integrated to industry.

The antenna efficiency depends on the deposit thickness and conductivity of the material. Efficiency of the copper based antenna is $92 \%$ for a thickness of 35 $\mu \mathrm{m}$. For a thickness of $6 \mu \mathrm{m}$, the copper antenna efficiency decreases to $37 \%$. The optimization of the PEDOT:PSS antenna structure permits it to reach an efficiency of $10 \%$ with PEDOT conducting product which lead to suitable radiation performance (Table 1).

Despite the fact that we have four thousand times less in conductivity, the low deposited thickness and a lower radiation efficiency, this antenna allows however a maximum read distance of $75 \mathrm{~cm}$ which is suit for most of traceability and medical applications. The antenna efficiency leads to a low gain and read distance compared to the copper antenna.

\section{Discussions}

The above results characterize materials in terms of radiation efficiency and radiolucency and highlight the PEDOT:PSS. These results allow us to correlate the image contrast, efficiency and the thickness of the material.

In Figure 9, contrast is calculated using Equation (1):

$$
C=\frac{\text { mean ROI }- \text { mean BACKGROUND }}{\text { mean BACKGROUND }}
$$

where mean ROI is the average of the pixel grey values in the region of interest and mean BACKGROUND is the average of the pixel grey values in the background of the image.

Calculating contrast requires consistent and sufficiently large regions of inter-

Table 1. Efficiency of antennas.

\begin{tabular}{cccc}
\hline Antenna type & Copper & Copper & PEDOT:PSS \\
\hline Thickness $(\mu \mathrm{m})$ & 35 & 6 & 6 \\
Conductivity $(\mathrm{S} / \mathrm{m})$ & $5.8 \times 10^{7}$ & $5.8 \times 10^{7}$ & $1.4 \times 10^{4}$ \\
Efficiency & $92 \%$ & $37 \%$ & $10 \%$ \\
\hline
\end{tabular}




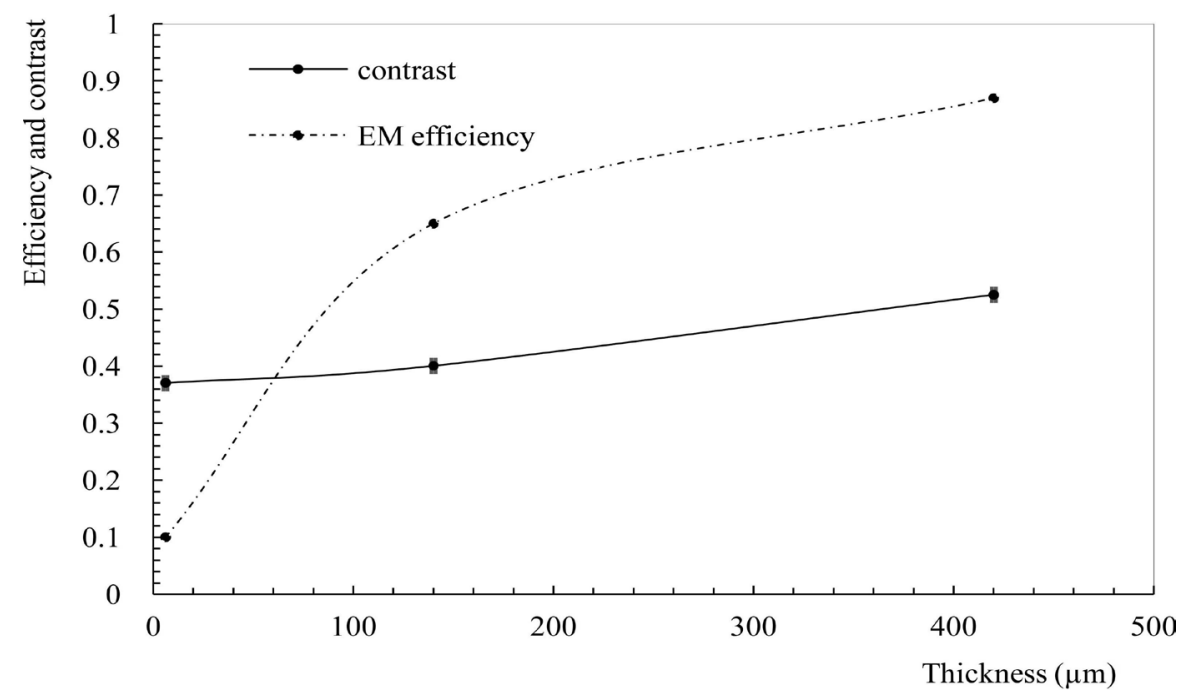

Figure 9. Efficiency and contrast in function of PEDOT:PSS thickness.

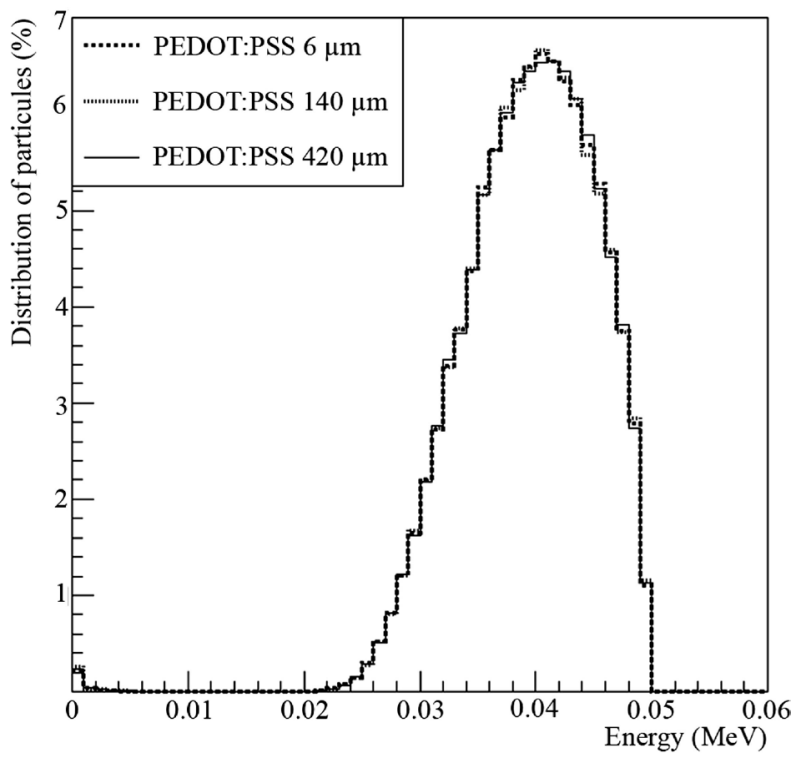

Figure 10. The impact of PEDOT:PSS on the beam quality.

est on the image to make it meaningful and define regions of interest. The antenna is of small size, larger objects like plates of specific thickness are used to obtain a coherent result. Three specific thicknesses are studied: 6 micrometers represent the current structure of our antenna, 140 micrometers is the skin depth associated to this antenna, 420 micrometers represents three times of the skin depth, antenna efficiency is then the highest. As seen above, we get an efficiency of $10 \%$ for the PEDOT antenna with $6 \mu \mathrm{m}$ thickness. Our model allows us to determine that the efficiency is $63 \%$ for the skin depth and almost reaches $90 \%$ for a thickness of $430 \mu \mathrm{m}$. In this thickness range of PEDOT, the contrast is low and slowly increases to reach 0.5 for a thickness of $430 \mu \mathrm{m}$. Thus, regardless of the thickness of PEDOT considered, the antenna remains radiolucent. Figure 10 finally shows that PEDOT antenna has no influence on the X-ray beam up to 
a thickness of $0.4 \mathrm{~mm}$.

Consequently, for the desired detection range, it is possible to determine the necessary efficiency and deduce the thickness of the PEDOT antenna. This process will minimize the amount of material to use when printing methods allow. Figure 9 finally shows the trade-off between radiation efficiency and contrast according to material thickness.

\section{Conclusions}

In this study, we have demonstrated that the radiolucency and radiation efficiency of a material may be estimated by our predictive model. Our model could be reused to optimize both radiolucent and Electro Magnetic characteristics for other antennas depending on the applications. This model for quantifying the radiolucency could also be used for the characterization of materials used for medical implants. Today radiopacity or radiolucency of implants is generally estimated once they have been implanted, usually visually according to the medical images and depending on the user. Using our simulations would give a new perspective to medical staff and reduce research cost from an industrial point of view. The potential of PEDOT: PSS as a radiolucent and radiating material has been explored and reveals a promising material for radiology. The reasonable read distance is suitable for wireless medical technologies; the radiolucency of PEDOT:PSS is adapted of the $\mathrm{x}$-rays used in medical imaging. The antenna proposed in this study could be combined with a sensor recording vital signs like temperature, blood pressure sensor or ECG electrodes. In this way, a complete wireless monitoring will be achievable on a daily basis, during imaging procedures or in the operating room.

\section{References}

[1] Akay, M., et al. (2012) Emerging Technologies for Patient-Specific Healthcare. IEEE Transactions on Information Technology in Biomedicine, 16, 185-189. https://doi.org/10.1109/TITB.2012.2187810

[2] Besson, M., Von Czettriz, G. and and Bax, R. (1999) Wireless Medical Diagnosis and Monitoring Equipment. Patent Application 5862803, USA.

[3] Khaddar, M.A., et al. (2012) Emerging Wireless Technologies in E-Health Trends, Challenges, and Framework Design Issues. International Conference on Multimedia Computing and Systems.

[4] Zheng, Y.L., et al. (2014) Unobtrusive Sensing and Wearable Devices for Health Informatics. IEEE Transactions on Biomedical Engineering, 61, 1538-1554. https://doi.org/10.1109/TBME.2014.2309951

[5] Kang Chang, R. (2014) EGC Leads System for Newborn ECG Screening. Patent Application 8369924B1, USA.

[6] Day, J.L. (1997) Radiolucent Head Support. Patent Application 5276927A, USA.

[7] Castagnola, V., et al. (2015) Parylene-Based Flexible Neural Probes with PEDOT Coated Surface for Brain Stimulation and Recording. Biosensors and Bioelectronics, Elsevier, 67, 450-457. https://doi.org/10.1016/j.bios.2014.09.004

[8] Liaparinos, P.F. (2010) Monte Carlo Simulation in Medical Imaging. E-Journal of 
Science and Technology, 2, 7-13.

[9] Philips Medical Systems Nederland. Philips AlluraXper FD20/10 and Allura FD20/ 20 Functional Description, 2008.

[10] Jan, S., et al. (2004) GATE: A Simulation Toolkit for PET and SPECT. Physics in Medicine and Biology, 49, 4543-4561. https://doi.org/10.1088/0031-9155/49/19/007

[11] Agostinelli, S., et al. (2003) Geant4-A Simulation Toolkit. Nuclear Instruments and Methods in Physics Research Section A: Accelerators, Spectrometers, Detectors and Associated Equipment, 506, 250-303. https://doi.org/10.1016/S0168-9002(03)01368-8

[12] Allison, J., et al. (2006) Geant4 Developments and Applications. IEEE Transactions on Nuclear Science, 53, 270-278. https://doi.org/10.1109/TNS.2006.869826

[13] Poludniowski, G. and Evans, P. (2007) Calculation of X-Ray Spectra Emerging from an X-Ray Tube. Part I. Electron Penetration characteristics in X-Ray Targets. Medical Physics, 34, 2164-2174. https://doi.org/10.1118/1.2734725

[14] Poludniowski, G. (2007) Calculation of X-Ray Spectra Emerging from an X-Ray Tube. Part II. X-ray Production and Filtration in X-Ray Targets. Medical Physics, 34, 2175-2186. https://doi.org/10.1118/1.2734726

[15] Poludniowski, G. (2009) SpekCalc: A Program to Calculate Photon Spectra from Tungsten Anode X-Ray Tubes. Physics in Medicine \& Biology, 54, 433-438. https://doi.org/10.1088/0031-9155/54/19/N01

[16] Camarasu-Pop, S., et al. (2013) Monte-Carlo Simulation on Heterogeneous Distributed Systems: A Computing Framework with Parallel Merging and Checkpointing Strategies. Future Generation Computer Systems, 29, 728-738.

https://doi.org/10.1016/j.future.2012.09.003

[17] Kao, T., et al. (2007) A Compensated Radiolucent Electrode Array for Combined EIT and Mammography. Physiological Measurement, 28, 291-299. https://doi.org/10.1088/0967-3334/28/7/S22

[18] Li, H., et al. (2016) In Vitro Investigation of NiTiW Shape Memory Alloy as Potential Biomaterial with Enhanced Radiopacity. Materials Science and Engineering $C$, 60, 554-559. https://doi.org/10.1016/j.msec.2015.12.006

[19] Glover, J.L., and Hudson, L.T. (2016) An Objectively-Analyzed Method for Measuring the Useful Penetration of X-Ray Imaging Systems. Measurement Science and Technology, 27, Article ID: 065402. https://doi.org/10.1088/0957-0233/27/6/065402 
Submit or recommend next manuscript to SCIRP and we will provide best service for you:

Accepting pre-submission inquiries through Email, Facebook, LinkedIn, Twitter, etc. A wide selection of journals (inclusive of 9 subjects, more than 200 journals)

Providing 24-hour high-quality service

User-friendly online submission system

Fair and swift peer-review system

Efficient typesetting and proofreading procedure

Display of the result of downloads and visits, as well as the number of cited articles Maximum dissemination of your research work

Submit your manuscript at: http://papersubmission.scirp.org/

Or contact ojopm@scirp.org 